\title{
What is the origin of chirality in the cholesteric phase of virus suspensions ?
}

\author{
Eric Grelet* and Seth Fraden \\ Department of Physics, MS-05\%, Brandeis University, Waltham, MA 02454, U.S.A.
}

(Dated: October 29, 2018)

\begin{abstract}
We report a study of the cholesteric phase in monodisperse suspensions of the rod-like virus $f d$ sterically stabilized with the polymer polyethylene glycol (PEG). After coating the virus with neutral polymers, the phase diagram and nematic order parameter of the $f d$-PEG system then become independent of ionic strength. Surprisingly, the $f d$-PEG suspensions not only continue to exhibit a cholesteric phase, which means that the grafted polymer does not screen all chiral interactions between rods, but paradoxically the cholesteric pitch of this sterically stabilized $f d$-PEG system varies with ionic strength. Furthermore, we observe that the cholesteric pitch decreases with increasing viral contour length, in contrast to theories which predict the opposite trend. Different models of the origin of chirality in colloidal liquid crystals are discussed.
\end{abstract}

Chirality leads to a wonderful variety of liquid crystalline phases [1], from the cholesteric state to very complex structures such as smectic blue phases [2]. In spite of its importance, the connection between chirality at the molecular scale and the macroscopic chiral structure of liquid crystalline phases is not yet understood, and quantitative prediction of the twist periodicity, also called cholesteric pitch, based on molecular features remains a unsolved problem. The elaboration of a rigorous statistical theory for the chiral interaction is one of the greatest challenges in the physics of liquid crystals.

We report and discuss experimental studies of the cholesteric phase of colloidal suspensions of filamentous bacteriophages, denoted $f d$. The rod-like virus $f d$ is a micron-length semi-flexible polyelectrolyte formed by a single stranded DNA around which coat proteins are helicoidally wrapped. Therefore $f d$ is a chiral object exhibiting a helical charge distribution. Suspensions of $f d$ rods in aqueous solution form several liquid crystalline phases with increasing concentration, and especially a cholesteric or chiral nematic phase which lies between the isotropic and smectic phases [3]. However, molecular chirality does not guaranty the existence of macroscopic chiral structure such as a cholesteric phase. For instance other chiral viruses such as Tobacco Mosaic Virus (TMV) [4], or Pf1 [5] with a helical structure extremely similar to $f d$, exhibit only a nematic phase. Up to now, theory has failed to answer even the simple qualitative question: When does macroscopic chirality, such as twist, occur in liquid crystals formed by helical molecules? In this Letter, by studying a well characterized system of rod-like virus stabilized by grafted neutral polymer, we highlight that counterions play a crucial role. More specifically we show that ionic strength is one of the major physical parameters controlling the chirality of colloidal liquid crystalline suspensions.

The bacteriophage $f d$ is a molecule with a contour length of $\mathrm{L}=880 \mathrm{~nm}$, a diameter of $D_{\text {bare }}=6.6 \mathrm{~nm}$, a

\footnotetext{
*Permanent address: Centre de Recherche Paul Pascal, UPR 8641,
} Avenue Albert Schweitzer, F-33600 Pessac, France. persistence length of $\xi=2200 \mathrm{~nm}$, a molecular weight of $\mathrm{M}_{W}=1.64 \times 10^{7} \mathrm{~g} / \mathrm{Mol}$, and a charge density of $10 \mathrm{e} / \mathrm{nm}$ in water at $\mathrm{pH}=8.1[\underline{5}]$. About 2700 copies of a single protein form the coat helicoidally wrapped following a 5 -fold rotation axis combined with an 2 -fold screw axis around the inner DNA [6]. The $f d$ virus was grown and purified following standard biological protocols [7]. To vary ionic strength (I), viruses were extensively dialyzed against a $20 \mathrm{mM}$ TRIS-HCl buffer at $\mathrm{pH}=8.1$ with an adjusted amount of $\mathrm{NaCl}$. All measurements were done at room temperature and the concentrations were determined using spectrophotometry with absorption coefficient of 3.84 $\mathrm{cm}^{2} / \mathrm{mg}$ at $269 \mathrm{~nm}$.

Although the $f d$ virus is charged in solution, it is possible to prepare sterically stabilized colloids by irreversibly binding neutral polyethylene glycol (PEG) of different molecular weights $(5,000$ or $20,000 \mathrm{~g} / \mathrm{Mol})$ to the surface of the virus [8]. End functionalized PEG molecules (Shearwater corp.) have been used to covalently attach the water soluble polymer to the amino groups on the coat proteins of $f d$ that are exposed to the solution. The refractive index increment, $\mathrm{dn} / \mathrm{dc}$, has been measured to estimate the degree of coverage of $f d$-PEG virus [9] , and $195 \pm 30$ polymers PEG-20k (400 \pm 40 for PEG-5k) are attached to each virus. This result corresponds to nearly complete coverage of the virus by the polymer. Indeed, by considering grafted polymers as spheres of radius of gyration $\mathrm{R}_{g}$, about $\pi\left(1+D_{\text {bare }} / 2 R_{g}\right) \times L / 2 R_{g}$ polymers can be close-packed around a virus of diameter $D_{\text {bare }}$ (Fig. 11). In the case of PEG-20K $\left(\mathrm{R}_{g}=7 \mathrm{~nm}[10]\right)$, this model predicts about 250 grafted polymers per virus, which is close to measured value.

The isotropic - cholesteric (I-N*) transition has been investigated for this system of $f d$ virus grafted with PEG [8]. According to Onsager [1], an isotropic to nematic phase transition occurs in suspensions of hard rods when the rod number density $n$ reaches $n \pi L^{2} D \simeq 16$ (eq. 1). This is experimentally observed for bare $f d$ (without any grafted polymer) over a wide range of ionic strengths as shown with square symbols in Fig. 11 Note that there is no difference in Onsager's theory between the isotropic-nematic and isotropic-cholesteric phase transitions. The free energy difference between the ne- 
matic and cholesteric phase is negligible compared to the free energy difference between the isotropic and nematic phase 12.

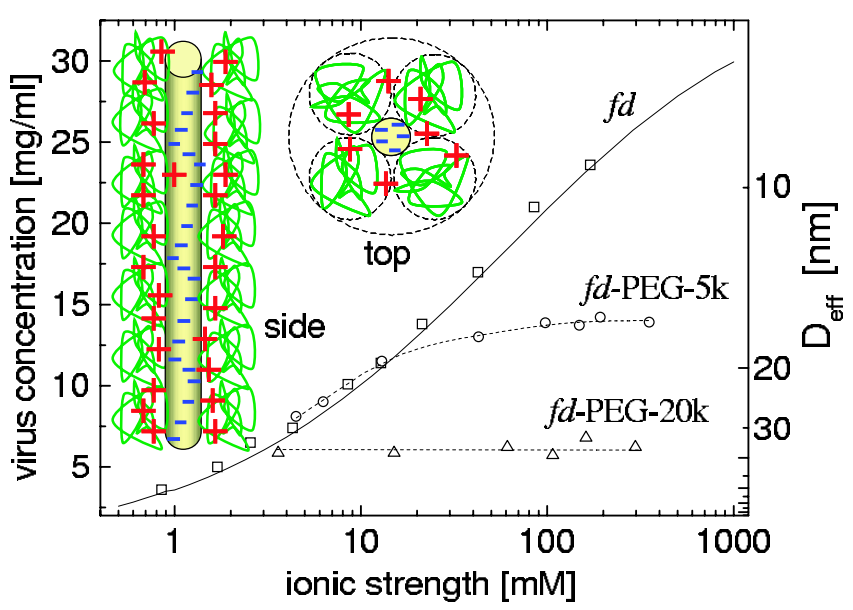

FIG. 1: Isotropic - cholesteric phase boundary as a function of ionic strength for bare virus $(f d)$ and for virus coated with neutral polymer ( $f d$-PEG-5k and $f d$-PEG-20k). The lines represent the highest concentration for which the isotropic phase, I, is stable (below line), whereas the range of the cholesteric phase, $\mathrm{N}^{*}$, is above each line. Squares and circles indicate the I-N* transition in $f d$ and in $f d$ coated with PEG-5k respectively, while triangles refer to the $f d$ virus coated with PEG-20k. The concentration of the $\mathrm{I}^{-\mathrm{N}^{*}}$ transition for bare $f d$ increases as a function of ionic strength as $1 / D_{\text {eff }}$ (solid line), where $D_{\text {eff }}$ is the effective diameter taking into account the electrostatic repulsion between rods at the I-N* transition [1]. $f d$ with grafted PEG shows identical behavior to nongrafted, up to an ionic strength corresponding to a $D_{\text {eff }}$ that matches the size of the stabilizing polymer layer. For a higher amount of added salt, the phase diagram becomes independent of ionic strength, and the $f d$-PEG system behaves as a sterically stabilized suspension. This is schematically represented by the cartoon of a virus with polymers grafted on its surface where $D_{\text {eff }}^{\text {electrostatic }}<D_{\text {eff }}^{\text {polymer }}$.

Because the $f d$ virus is charged, it is necessary to account for electrostatic repulsion by considering the colloids as having an effective diameter $\left(D_{\text {eff }}\right)$ larger than their bare diameter $\left(D_{\text {bare }}\right)$ with $D_{\text {eff }}$ roughly proportional to the Debye screening length [1]. $D_{\text {eff }}$ therefore decreases with increasing ionic strength 13] and approaches $D_{\text {bare }}$ at high ionic strength. By substituting $D_{\text {bare }}$ with $D_{\text {eff }}$ in equation (1), it follows that the concentration of $f d$ at the I-N* transition is inversely proportional to $D_{\text {eff. }}$. For $f d$-PEG rods, there is an ionic strength at which the coexistence concentrations of the I-N* transition become independent of ionic strength. The crossover from the regime where the rods are electrostatically stabilized to where they are sterically stabilized by "soft" repulsion between virus-bound polymers is shown in Fig. 10 This corresponds to a ionic strength of $\mathrm{I}=3 \mathrm{mM}$ for $f d-\mathrm{PEG}-20 \mathrm{k}$ and $\mathrm{I}=20 \mathrm{mM}$ for $f d$-PEG-5k. At this ionic strength, the diameter associated with electrostatic interactions $\left(D_{\text {eff }}\right)$ equals the diameter of the virus plus grafted polymer. It is therefore possible to extract an effective diameter for the polymer coated virus. In this range of ionic strengths, between 15 and $200 \mathrm{mM}$, the polymer is in a good solvent and the effects of $\mathrm{NaCl}$ on PEG properties are negligible in terms of radius of gyration and viscosity [14]. From Fig. [1 the effective diameter of $f d$-PEG-20k at $\mathrm{I}=3 \mathrm{mM}$ is $37 \mathrm{~nm}$, which is approximately equal to the hard diameter of $f d\left(D_{\mathrm{bare}}=6.6 \mathrm{~nm}\right)$ plus twice the diameter of PEG $\left(R_{g}=7 \mathrm{~nm}[10]\right): D_{\text {bare }}+4 R_{g}=35 \mathrm{~nm}$. The $f d-\mathrm{PEG}-5 \mathrm{k}$ complex has $D_{\text {eff }}=17 \mathrm{~nm}$ at high ionic strength, while $D_{\text {bare }}+4 R_{g}=19 \mathrm{~nm}\left(R_{g}=3 \mathrm{~nm}\right.$ for PEG-5k [10]).

The good agreement between the measured and estimated value of the $f d$-PEG effective diameter supports the model of polymer behaving mainly as a sphere attached to the surface of the virus. Thus, the independence of the phase diagram on ionic strength reported in Fig. 1 shows that $f d$ virus coated with PEG-20k is a system whose stability is entirely determined by polymer repulsion. This result has been confirmed by measuring the nematic order parameter of $f d$-PEG-20k close to the I-N* transition. Fig. 2 indicates that the order parameter, determined by birefringence under magnetic field, is independent of ionic strength just as is the phase diagram [15]. Therefore, $f d$-PEG-20k liquid crystal turns out to be a sterically stabilized system, that, surprisingly, continues to exhibit a cholesteric phase as shown in Fig. 3 Indeed, one could expect that grafted polymers on virus surface would screen chiral interactions, leading thus only to a nematic phase. To our knowledge, $f d$ virus coated with PEG is the first completely sterically stabilized system which still has chiral features.



FIG. 2: Ionic strength dependence of the nematic order parameter of $f d$ virus coated with PEG-20k measured close to the I-N* transition (samples concentrations around $\mathrm{c} \sim 9$ $\mathrm{mg} / \mathrm{ml}$ ). The order parameter is obtained from birefringence measurements after unwinding the cholesteric phase with a magnetic field. The dashed line is a guide for the eyes.

The insert of Fig. 3 presents a typical "fingerprint" texture of $f d$-PEG suspensions characteristic of a cholesteric phase observed by optical microscopy between crossed polarizers. After a few days of equilibra- 


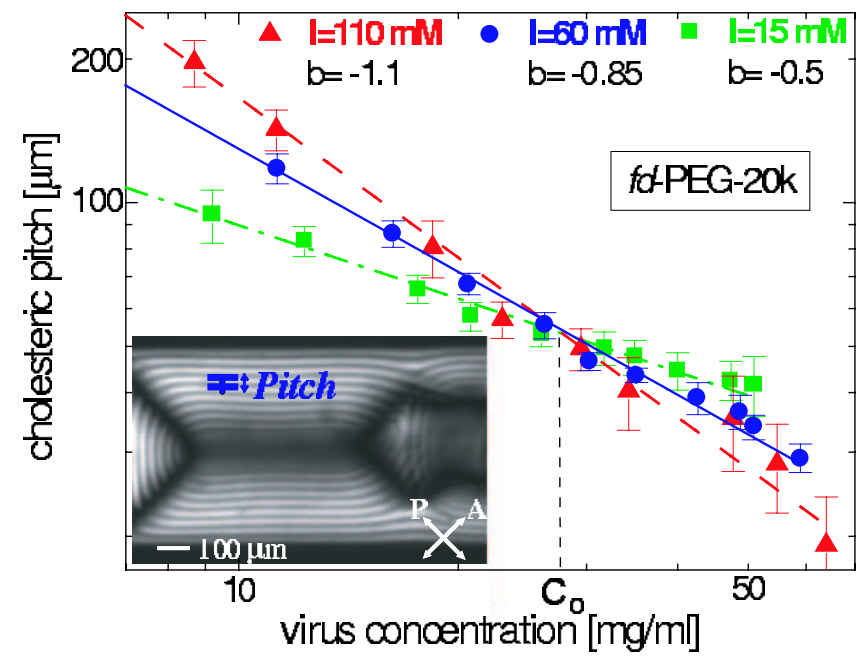

FIG. 3: Evolution of the cholesteric pitch (P) with the concentration (c) of $f d$ virus grafted with PEG-20k, for three different ionic strengths $(\mathrm{I}=15,60$, and $110 \mathrm{mM})$. Cholesteric pitch was measured from the "fingerprint" texture, as shown in the insert. The sample is contained in a cylindrical glass capillary of diameter $0.7 \mathrm{~mm}$ and several $\mathrm{cm}$ long. Error bars have been obtained by repeating the same measurement along the full length of the sample. The scaling exponent of the fit to $P \propto c^{b}$ is indicated for each ionic strength.

tion following the preparation of the samples, we have measured the dependence of cholesteric pitch on concentration and ionic strength (Fig. 31). Paradoxically, $f d-$ PEG-20k liquid crystals have a cholesteric pitch which depends on ionic strength. Close to the I-N* transition where both the phase diagram and order parameter are independent of ionic strength (Figs. [1 and 21), the pitch varies by a factor 2 from $100 \mu \mathrm{m}$ at $\mathrm{I}=15$ $\mathrm{mM}$ to $200 \mu \mathrm{m}$ at $\mathrm{I}=110 \mathrm{mM}$. In this range of low rod concentrations the mean interparticle distance $\left(d_{\text {inter }}\right)$ is about $60 \mathrm{~nm}$ : the $f d$-PEG surfaces are then separated by typically 3 bare diameters 15 . The corresponding volume fraction at the $\mathrm{I}-\mathrm{N}^{*}$ transition is $\Phi=0.2$ where $\Phi$ is defined by $\Phi=c N_{A} L \pi\left(D_{\text {eff }} / 2\right)^{2} / M_{W}$ with $N_{A}$ being the Avogadro's number. We can then calculate the concentration $\left(\mathrm{c}^{*}\right)$ for which the grafted polymers of $f d$ PEG-20k begin to overlap (corresponding to $\Phi^{*}=1$ ): this gives $\mathrm{c}^{*} \simeq 35 \mathrm{mg} / \mathrm{ml}$, which is close to the concentration $\mathrm{c}_{0}=28 \mathrm{mg} / \mathrm{ml}$ where the pitch is independent of ionic strength (Fig. 3). The concentration $c^{*}$ separates two regimes: we mainly focus on the dilute regime $\left(c<c^{*}\right)$, where chiral rods do not overlap. Complexity increases for $\mathrm{c}>\mathrm{c}^{*}$ because of short-range interactions between the polymers as well as electrostatic interactions between the viruses, which can induce intricate behaviors similar to those seen for instance in DNA condensation [16].

What then is the mechanism of transmission of chiral interactions between sterically stabilized viruses? The first model of the origin of intermolecular twist was proposed by Straley who considered two screw-like molecules with excluded-volume interactions [17]. He predicted the pitch to be independent of the concentration, which is not observed experimentally. Moreover, if chirality is transmitted by some microscopic detail of the virus, we then expect that the chiral effects will be diminished for $f d$ grafted with polymers. However, the same range of pitch has been seen for the $f d$-PEG system as for bare $f d$ (between 10 and $200 \mu \mathrm{m}$ ) [5]. That seems to exclude the strict steric model of packing of screws, because the thread of the screws of $f d$ virus is covered by a $30 \mathrm{~nm}$ thick polymer coat. Even with the assumption that the polymers grafted to the helical protein coat replicate the chiral symmetry of the underlying bare virus, it does not explain why the cholesteric pitch should depend on ionic strength.

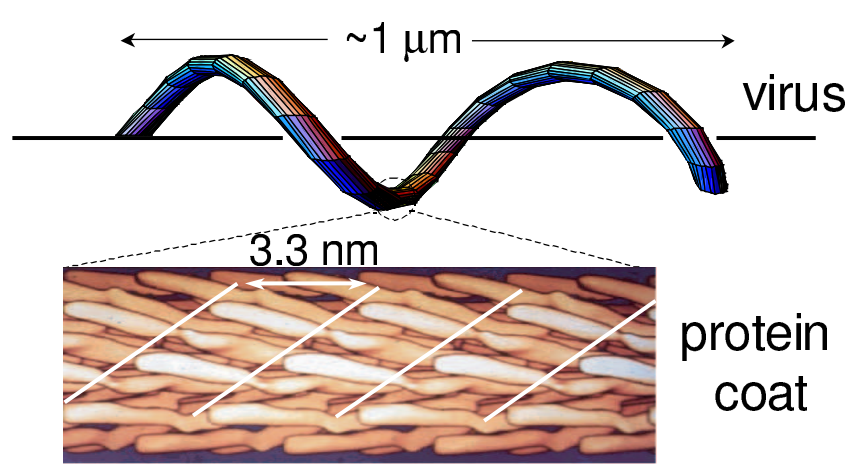

FIG. 4: Schematic representation of the "cork-screw" model: the chirality is transmitted between viruses by the helical shape (accentuated for illustration) of the whole virus at the micron length scale and not by the helical structure of the protein coat.

If the chirality is not transmitted at the microscopic length scale of the helical periodicity of the protein coat (with an axial repeat of $3.3 \mathrm{~nm}$ for $f d$ virus [6]), the existence of a superhelical twist, where chirality occurs at a much larger length scale, may be proposed. Brownian motion causes the virus to bend back and forth, and due to the coupling between flexibility and the underlying structural chirality imposed by the helical arrangement of coat proteins, the virus could twist more in one direction than in another leading to the creation of a "corkscrew" shape with a helical pitch of order a micron. A test [suggested by F. Amblard] of this model would be to measure the distribution of angles between the tangent vectors of the ends of a single virus confined to a surface. This is a Gaussian distribution for the worm-like chain model, but will have a maximum at a non-zero angle for a bent molecule, such as a cork-screw of length less than one full pitch. However, the "cork-screw" model can not be the whole story as Pf1, a chiral and flexible virus with a structure very similar to fd, has only a nematic phase, which implies that not all chiral flexible molecules form cork-screws. Additionally, completely rigid rods like cellulose [18] and chitin [19] form a cholesteric phase implying that the cork-screw distortion is not necessary for cholesteric production. An interesting experiment would 
be to coat these rigid molecules with long polymer. If they still form the cholesteric phase then the cork-screw model would be rejected.

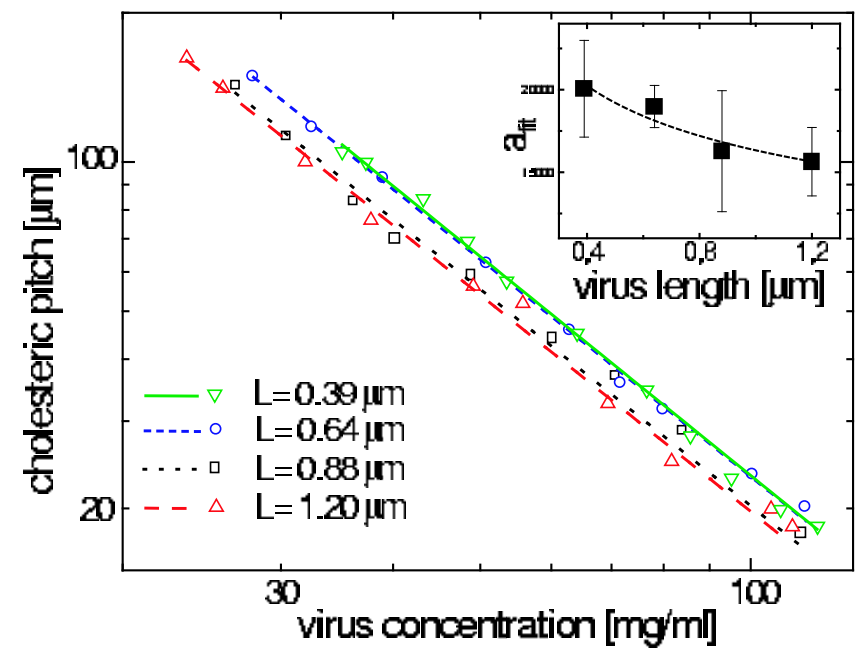

FIG. 5: Graph of the cholesteric pitch $(\mathrm{P})$ versus the concentration (c) for mutant viruses of different contour lengths (L). The ionic strength is $60 \mathrm{mM}$. The result of the fit $P=a_{f i t} c^{b}$ indicates the same scaling exponent $b=-1.45$ for all mutants. The insert indicates the dependence of the pitch with the length of the virus at a given concentration: $P \propto a_{f i t} \propto$ $1 / L^{0.25}$. This result contradicts the main existing theories of chirality in liquid crystals which predict $\left.P \propto L^{2}[20] 21\right]$.

One of the most advanced theories of cholesteric assemblies of charged rods has been proposed by Kornyshev et al. for DNA. They suggest that various details of the helical symmetry of macromolecules surface charge pattern may be responsible for macroscopic twist [20]. The main idea is based on direct chiral electrostatic interaction be- tween long helical molecules. Their attempt is promising, but how can such a theory account, even qualitatively, for our results on $f d$ virus stabilized with polymer? Moreover, the prediction of a cholesteric pitch increasing with the square of the viral contour length $\left(P \propto L^{2}\right)$ shown both by Kornyshev et al. [20] and by Harris et al. [21] is in contradiction to our results obtained with mutant viruses (without any polymer grafted). Indeed, we are able to grow monodisperse mutants 8] where only the contour length varies, keeping unchanged the local structure of the viruses 22 . We find that the cholesteric pitch decreases with increasing viral contour length as shown in Fig. 5] These experiments suggest that current theories do not capture all the essential elements of the connection between microscopic and macroscopic chirality in colloidal liquid crystals.

In conclusion, we have presented in this Letter a system of chiral colloidal rods coated with neutral polymer, which behaves as a sterically stabilized suspension because the phase diagram and order parameter are independent of ionic strength. However, this liquid crystal has the baffling feature that it exhibits a cholesteric phase whose pitch depends on ionic strength. If the cholesteric phase is induced by the charges on the the virus which are buried by a polymer coat of thickness corresponding to several Debye screening lengths, then we conclude that the cholesteric phase arises from very weak and subtle forces. Our result highlights the fundamental role of counterions in the existence of a macroscopic chirality and should stimulate new theoretical investigations on this fundamental topic of the origin of chirality in liquid crystalline phases.

This research was supported by NSF/DMR grants. We acknowledge Kirstin Purdy for her assistance in the preparation of virus mutants.
[1] P.G. de Gennes and J. Prost, The Physics of Liquid Crystals (Clarendon, Oxford, 1993).

[2] E. Grelet et al., Phys. Rev. E 65, 050701 (2002).

[3] Z. Dogic and S. Fraden, Phys. Rev. Lett. 78, 2417 (1997).

[4] F.C. Bawden, N.W. Pirie, J.D. Bernal, and I. Fankuchen, Nature 138, 1051 (1936).

[5] Z. Dogic and S. Fraden, Langmuir 16, 7820 (2000).

[6] S. Bhattacharjee, M.J. Glucksman, and L. Makowski, Biophys. J. 61, 725 (1992).

[7] J. Sambrook, E.F. Fritsch, and T. Maniatis, Molecular cloning (Cold Spring Harbor Laboratory Press, NewYork, 1989).

[8] Z. Dogic and S. Fraden, Phil. Trans. R. Soc. Lond. A 359, 997 (2001).

[9] The dn/dc of PEG, $f d$, and $f d$-PEG complex were measured separately. The concentration of virus in $f d$-PEG suspensions is determined by UV absorption (The PEG does not absorb in the UV). Since $\mathrm{dn} / \mathrm{dc}$ is proportional to the mass density, the $\mathrm{dn} / \mathrm{dc}$ difference between the bare virus and the complex is due to grafted polymer.
[10] K. Devanand and J.C. Selser, Macromolecules 24, 5943 (1991).

[11] L. Onsager, Ann. N.Y. Acad. Sci 51, 627 (1949).

[12] A. Saupe, Angew. Chem. Int. Ed. 7, 97 (1968).

[13] J. Tang and S. Fraden, Liq. Cryst. 19, 459 (1995).

[14] F.E. Bailey, Jr., and R.W. Callard, J. Appl. Polymer Sci. 1, 56 (1959).

[15] K.R. Purdy et al., arXiv:cond-mat/0210030 (2002).

[16] W.M. Gelbart et al., Phys. Today 53, 38 (2000).

[17] J.P. Straley, Phys. Rev. A 14, 1835 (1976).

[18] J.-F. Revol et al., Int. J. Bio. Macromol. 14, 170 (1992); L. Heux, G. Chauve, and C. Bonini, Langmuir 16, 8210 (2000); J. Araki, M. Wada, and S. Kuga, Langmuir 17, 21 (2001).

[19] J.-F. Revol and R.H. Marchessault, Int. J. Bio. Macromol. 15, 329 (1993).

[20] A.A. Kornyshev, S. Leikin, and S.V. Malinin, Eur. Phys. J. E 7, 83 (2002).

[21] A.B. Harris, R.D. Kamien, and T.C. Lubensky, Phys. Rev. Lett. 78, 1476 (1997). 
[22] K.R. Purdy, E. Grelet, and S. Fraden (unpublished). 